\title{
Long-term stability after orthodontic treatment remains inconclusive
}

\author{
What are the long-term effects of orthodontic treatment on morphologic \\ stability and patient satisfaction?
}

\author{
Bondemark L, Holm AK, Hansen K, et al. \\ Long-term stability of orthodontic treatment and patient \\ satisfaction. Angle Orthod 2007; 77:181-191
}

\begin{abstract}
Data sources PubMed and Cochrane Library electronic databases were searched from 1966 to January 2005. Reference lists of the relevant studies were searched by hand.

Study selection For inclusion, articles had to satisfy the following criteria: have a follow-up period of at least 5 years post-retention; report on randomised clinical trials (RCT), prospective or retrospective clinical controlled studies or on cohort studies; and investigate orthodontic treatment including fixed or removable appliances, selective grinding or extractions.
\end{abstract}

Data extraction and synthesis Screening of eligible studies, data extraction and assessment of methodological quality was conducted independently and in duplicate. The level of evidence of the included studies was graded as high, moderate or low according to predetermined criteria.

Results The search strategy resulted in 1004 abstracts or full-text articles, of which 38 met the inclusion criteria. Treatment of crowding resulted in successful dental alignment but the mandibular arch length and intercanine width gradually decreased, and crowding of the lower anterior teeth reoccurred post-retention. This condition was unpredictable at the individual level. Treatment of Angle class-II division-1 malocclusion with a Herbst appliance normalised the occlusion. Relapse occurred but could not be predicted at the individual level. The scientific evidence was insufficient to draw conclusions about treatment of cross-bite, angle class III, open-bite and various other malocclusions, or about long-term patient satisfaction.

Conclusions Despite a large number of studies on long-term stability after orthodontic treatment, this systematic review shows that evidence-based conclusions are few. This is mostly because of inherent problems with retrospective and inferior study design. There is a great need for well-designed prospective studies with untreated control groups; sufficient sample sizes; and sample selection according to type of malocclusion, age and growth pattern.
Address for correspondence: Lars Bondemark, Faculty of Odontology, Malmö University, Department of Orthodontics, Cal Gustavs väg 34, Malmö, Scania SE20506 Malmö, Sweden. E-mail: lars.bondemark@od.mah.se

\section{Commentary}

For a patient who has undergone orthodontic treatment, which normally takes between 18 and 24 months and may have involved dental extractions, relapse of the final result is a great disappointment. This ambitious study aims to evaluate stability of orthodontic treatment, as well as patient satisfaction, for patients followed up for at least 5 years from the end of the treatment. This study was commissioned by the Swedish Council on Technology Assessment and Healthcare, presumably to assess the long-term health benefits of orthodontic treatment.

The literature search encompassed almost 40 years of articles. An information specialist rather than an orthodontist performed this, and it seems that a fairly limited number of MESH ( Medical Sunject Headings) terms were employed. The reference lists of articles discovered during the electronic search were also used to find further studies.

Of the 1004 studies identified (and a further 19 found from the reference list of the initial group), only 38 fulfilled the inclusion criteria. Of these, none was sufficiently robust to be graded as providing the best-quality evidence (grade $\mathrm{A}$ ). The remainder were split almost equally, into 20 grade $\mathrm{B}$ and 18 grade $\mathrm{C}$ studies and were also divided into eight treatment-subgroups, as follows:

- Treatment of crowding

- Treatment of class II malocclusion

- Treatment of crossbite

- Treatment of class III malocclusion

- Treatment of open-bite

- Treatment of various other malocclusions

- Patient satisfaction

Generally, there were too few studies in most of these subgroups for the authors to draw any evidence-based conclusions. Only the subgroups of studies looking at treatment of crowding and at treatment of class II malocclusion yielded meaningful results. In the former set, the eight studies that met grade-B criteria were all retrospective and their results indicated that: arch length and intercanine width of the mandible were reduced and crowding of the mandibular incisors frequently recurred during the follow-up period. As a result of this, many of the original authors advocated use of lifelong bonded mandibular canine retainers to retain a stable result.

The argument about whether we should use retainers long-term or not is effectively resolved - most orthodontists will warn patients at the beginning of treatment to expect lifelong retention should they wish their teeth to remain stable. The main debate is now about which retention regimen is most effective. Many orthodontists prefer the use of removable retainers, which can be controlled by the patient and do not necessarily need regular follow-up checks. Indeed, if a removable retainer breaks, the patient will know immediately and it is usually straightforward and relatively inexpensive to have 


\section{ORTHODONTICS}

them replaced. Tooth movements can occur within removable retainers, however, which may be unacceptable to the patient. The patient often chooses not to continue wearing their removable retainers and some then relapse.

Fixed retainers are out of the patient's control and, in general, prevent those teeth to which they are affixed from moving. There may be problems maintaining excellent oral hygiene with these, though, and flossing is particularly challenging. Fixed retainers can break and this is not always noticed by the patient until a tooth starts to move off-line. By this stage, recovery of the tooth may require some retreatment. Of more concern are anecdotal reports of teeth being moved during retention by twisted flexible wire retainers that have become active. It is therefore advisable that fixed retainers are regularly checked, either by the orthodontist or the patient's general practitioner: this involves time and cost.

In the treatment of class II malocclusion subgroup, 15 studies were identified, but only six of these even rated grade B. The majority of these studies involved the Herbst appliance. The conclusions drawn were that the Herbst appliance normalised the dentition and occlusion to class I and also that relapse occurred but could not be predicted at the individual level. It is interesting that the majority of the well-designed class II treatment studies in this report employed the Herbst device. This is an idiosyncratic fixed functional appliance, which maintains the mandible in a protruded position using fixed upper and lower elements, connected by a sliding tube and piston mechanism on each side. In the UK, very few practitioners use the
Herbst appliance although it has been reported in one RCT carried out by the Manchester group. ${ }^{1}$ Most UK practitioners use removable functional appliances, the most common of which is the twin block. Extrapolating the results of the Herbst studies to other treatment modalities will therefore require caution.

Although this study aimed to investigate patient satisfaction following orthodontic treatment, the authors felt that no evidencebased conclusions were possible because of the small number of studies found. They were astonished to find that only a few studies measured patient satisfaction over the long-term. They therefore concluded future investigations in this area are much-needed. The final conclusion was that, despite a large number of studies of long-term stability after orthodontic treatment, few evidence-based conclusions can be drawn. Once again, in order to provide answers, further welldesigned prospective studies with untreated control groups, adequate sample size and sample selection according to the type of malocclusion, age and growth pattern are required.

\section{Julian O'Neill}

Orthodontic Department, Kettering General Hospital NHS Trust, Kettering, Northamptonshire, UK

1. O'Brien K, Wright J, Conboy F, et al. Effectiveness of treatment for class II malocclusion with the Herbst or twin-block appliances: a randomized, controlled trial. Am J Orthod Dentofacial Orthop 2003; 124:128-137.

Evidence-Based Dentistry (2007) 8, 5-6. doi:10.1038/sj.ebd.6400511 